\title{
Novel Conjugated Polymer Containing Aromatic Ring and Selenium in Backbone. Kinetics of the Addition Reaction as the Model for Addition Polymerization
}

\author{
Nobuki Metaka, Eiichi Kobayashi, ${ }^{*}$ Sadahito Aoshima, \\ and Junji FuruKawA \\ Department of Industrial Chemistry, Faculty of Science and Technology, \\ Science University of Tokyo, Noda, Chiba 278, Japan
}

(Received August 5, 1994)

\begin{abstract}
The addition reaction mechanism of benzeneselenol to ethynylbenzene was studied as a model for the addition polymerization (so called polyaddition) of 1,4-benzenediselenol to 1,4-diethynylbenzene. The addition reaction occurred without an appreciable side reaction in the presence of AIBN and an adduct of the anti-Markownikoff's structure ( $(0,-\mathrm{Se}-\mathrm{CH}=\mathrm{CH}-0)$ was quantitatively obtained. The addition reaction is very similar to the addition polymerization of 1,4-benzenediselenol to 1,4-diethynylbenzene from the point of the geometrical structure of the polymer chain. A kinetic study showed that a rate-determining step of the addition reaction was the addition step of the benzeneseleno radical to ethynylbenzene. The results were compared with those of the addition reaction of benzenethiol to ethynylbenzene. By the competitive addition reaction, ethynylbenzene had 80 times higher reactivity than styrene toward benzeneselenol at $60^{\circ} \mathrm{C}$ in benzene.
\end{abstract}

KEY WORDS Addition Polymerization / Addition Reaction / Reaction Mechanism / Kinetics / Ethynylbenzene / Benzeneselenol / SeleniumContaining Polymer/ $\beta$-Phenylselenostyrene $/{ }^{13} \mathrm{C}$ NMR / Competitive Addition Reaction /

Recently, organoselenium compounds such as tetramethyltetraselenofulvalene perchlorate complex and related compounds have attracted attention as organic superconductors. ${ }^{1}$ However, the syntheses of conjugated polymers containing selenium are scarcely known. Sandman et al. reported the synthesis of poly ( $p$-phenyleneselenide) by polycondensation of $p$-dibromobenzene and sodium selenide. ${ }^{2}$ Druy et al. prepared polyselenophene by electropolymerization of selenophene. ${ }^{3}$

The authors reported studies on the conjugated polymer containing sulfur by addition polymerization (so called polyaddition) of dithiol monomers to diethynyl monomers. ${ }^{4-6}$ For example, the addition polymerization of

\footnotetext{
* To whom all correspondence should be addressed.
}

1,4-benzenedithiol (BDT) to 1,4-diethynylbenzene (DEB) proceeded rapidly in solution ${ }^{4}$ or in solid state ${ }^{7}$ under UV-irradiation. A conjugated linear polymer of the antiMarkownikoff's structure was obtained. The thin polymer film obtained by the solid state polymerization had a layer structure. The layer structure of polymer films increased or decreased reversibly by irradiation of the characteristic light. $^{8}$ In the previous paper, ${ }^{9}$ the authors investigated the synthesis of the conjugated polymer containing selenium by the addition polymerization of 1,4-benzenediselenol (BDSe) to DEB as shown in eq 1. The polymerization of BDSe to DEB was carried out by UV-irradiation in toluene at $60^{\circ} \mathrm{C}$ under 
nitrogen atmosphere. The polymerization proceeded at such a fast rate as to give $60-70 \%$ yield in several minutes. A pale-yellowish poly$\operatorname{mer}\left(\bar{M}_{n}=20000-30000\right)$ precipitated with the polymerization and had an anti-Markownikoff's structure. The polymer containing selenium exhibited electrical conductivity as $10^{-5} \mathrm{~S} \mathrm{~cm}^{-1}$ on doping with iodine. The solid state polymerization ${ }^{10}$ of BDSe to DEB was also found to yield a polymer film with a layer structure as well as BDT to DEB. ${ }^{7}$ However, the conjugated polymers did not dissolve in conventional organic solvents and the polymerization mechanism is also unclear.

In this article, to study the polymerization mechanism and polymer structure of BDSe and $\mathrm{DEB}$, a model addition reaction of benzeneselenol (BSe) to ethynylbenzene (EB) was investigated mainly by a kinetic method (eq 2). The results were compared with the addition reaction of benzenethiol (BT) to $\mathrm{EB}^{11}$ and $\mathrm{BSe}$ to styrene $(\mathrm{St}){ }^{12}$<smiles>C#Cc1ccc(C=CC=Cc2ccc(C=Cc3ccc(S(=O)(=O)C=Cc4ccccc4)cc3)cc2)cc1</smiles>

\section{EXPERIMENTAL}

\section{Reagent}

BSe was prepared according to the literature. $^{13}$ BT, EB, and St (Tokyo Kasei Kogyo Co. each) were obtained commercially. These reagents were purified three times by vaccum distillation under nitrogen atmosphere before use. Their purities were confirmed to be over $99 \%$ by GC. Benzene and toluene (Nacalai Tesque Inc. each) as reaction solvents were distilled over calcium hydride and degassed to exclude oxygen under reduced pressure for $20 \mathrm{~min}$ before use. 2,2'-Azobisisobutyronitrile (AIBN; Wako Pure Chem. Ind. Ltd.) was recrystallized from methanol. nHexadecane (Tokyo Kasei Kogyo Co.) as an internal reference for GC was purified by vaccum distillation under nitrogen atmosphere. Diphenyldiselenide was prepared by the dimerization of BSe under air. Nitrogen gas $(99.99 \%)$ was purified as shown in the previous paper. ${ }^{11}$

\section{Kinetics of Addition Reaction}

In a two-necked flask with a three-way stopcock and a septum cap, each benzene solution of EB, BSe, AIBN, and $n$-hexadecane was prepared by a semi-closed method using a stainless pipe syhpon method under precisely purified nitrogen atmosphere. ${ }^{11}$ The reaction was carried out by introducing each solution into a reaction vessel by the syphon method. The reaction temperature was maintained mainly at $65^{\circ} \mathrm{C}$. The course of the reaction was monitored every 3 min with measurement of the concentration of the adduct by GC.

\section{Measurements}

For characterization of the reagents and adduct and for monitoring the course of the reaction, the following instruments were used: ${ }^{1} \mathrm{H}$ NMR: JEOL PMX-60si and JEOL JNM-EX400; ${ }^{13} \mathrm{C}$ NMR: JEOL JNM-EX400; IR: Hitachi 260-50 spectrophotometer. A molecular weight distribution (MWD) curve was measured by a gel permeation chromatography (GPC) in chloroform on a JASCO TRI ROTAR instrument equipped with a polystyrene gel (Shodex K-2002; inner diameter $20 \mathrm{~mm}$, length $300 \mathrm{~mm}$ ) and a refractive index detector. Gas chromatograms (GC) were measured on a Hitachi GC 163 equipped with a thermal conductivity detector (TCD) and a Silicone GE SE-30 column $(2 \mathrm{~m})$ at $250^{\circ} \mathrm{C}$ under hydrogen carrier gas.

\section{RESULTS AND DISCUSSION}

\section{Addition Reaction of BSe to $E B$}

i) Influence of a Trace Amount of Oxygen. The addition reaction mechanism of BSe and EB was investigated by a kinetic method. Initial rates were determined from time-yield curves 
of the adduct formation. Since oxygen reacts with $\mathrm{BSe}$ to give benzeneseleno radical $^{14}$ similar to the case of BT, ${ }^{15}$ a trace amount of oxygen remaining in the reaction vessel promotes the addition reaction of $\mathrm{BSe}$ to $\mathrm{EB}$ at the early stage of the reaction. To keep a constant concentration of a trace amount of oxygen in the vessel, a mixture of BSe, EB, and AIBN solutions was prepared by a semi-closed method using a stainless pipe syphon not by a medical syringe under nitrogen atmosphere, while nitrogen gas $(99.99 \%)$ was purified through glass columns containing activated copper supported on kieselguhr at $190^{\circ} \mathrm{C}$, anhydrous calcium chloride, silica gel, and Molecular Sieves 4A in this order. However, reproducibility of the initial rate was not obtained under the purified nitrogen atmosphere. A representative example is shown in Figure 1 . AIBN and a trace amount of oxygen react with $\mathrm{BSe}$ to give benzeneseleno radical (eq 3).

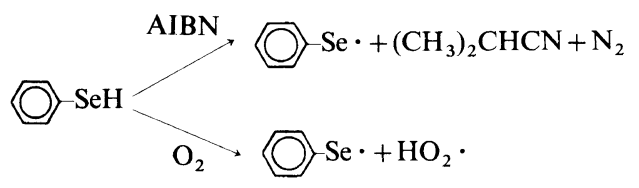

Nitrogen gas must therefore be purified strictly. Nitrogen gas purified as above described was further purified through a glass column containing Molecular Sieves 4A at near liquid nitrogen temperature. The time-yield curve of the adduct formation (Figure $1 \bigcirc$ ) under highly purified nitrogen can be extrapolated to the origin with good reproducibility. The addition reaction of BSe to EB was consequently found to be very sensitive toward oxygen. A kinetic study of this reaction should be carried out under highly purified nitrogen.

ii) Preparation of Addition Product of BSe and $E B$. The addition reaction of $\mathrm{BSe}$ $\left(1.0 \mathrm{~mol}^{-1}\right)$ to $\mathrm{EB}\left(1.0 \mathrm{~mol}^{-1}\right)$ was carried out with an AIBN initiator $\left(0.01 \mathrm{~mol}^{-1}\right)$ at $60^{\circ} \mathrm{C}$ in benzene under nitrogen atmosphere. The addition reaction was completed in $2 \mathrm{~h}$.

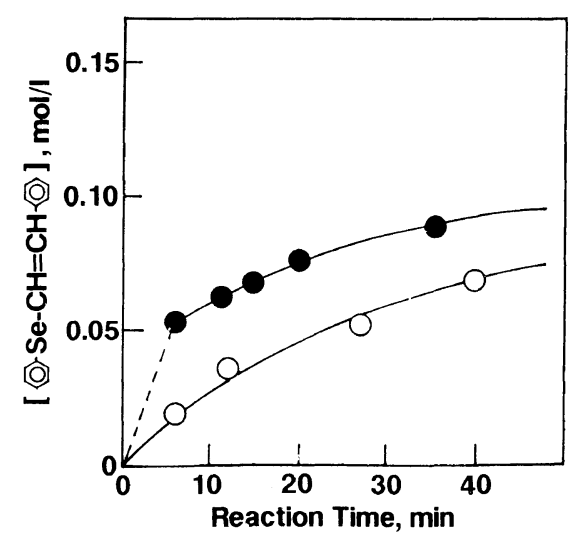

Figure 1. Effects of oxygen on the adduct formation of $\mathrm{BSe}$ and $\mathrm{EB}$ at $40^{\circ} \mathrm{C}$ in the dark under nitrogen atmosphere. $[\mathrm{EB}]_{0}=[\mathrm{BSe}]_{0}=3.8 \times 10^{-1} \mathrm{moll}^{-1},[\mathrm{AIBN}]_{0}=$ $3.8 \times 10^{-3} \mathrm{moll}^{-1}$ in benzene. $\bigcirc$, four nine nitrogen gas was purified through a glass column containing reduced copper; $\bigcirc$, purified four nine nitrogen was further purified through a glass column containing Molecular Sieves 4A at near liquid nitrogen temperature.

The crude product obtained by removal of the benzene from the reaction mixture without pretreatment was a slightly yellowish transparent liquid.

The crude product was analyzed by GPC, GC, ${ }^{1} \mathrm{H}$ NMR, and ${ }^{13} \mathrm{C}$ NMR. Over $99 \%$ of the product was found to be the $1: 1$ adduct of BSe and EB. No by-products such as oligomers of EB were found by GPC (Figure 2). Diphenyldiselenide, if formed by a coupling reaction of benzeneseleno radicals, could not be confirmed by GPC, GC, and ${ }^{1} \mathrm{H}$ NMR analyses because of the overlap of these spectra with the $1: 1$ adduct. Therefore, analysis by ${ }^{13} \mathrm{C}$ NMR spectroscopy (Figure 3) was done, but diphenyldiselenide was hardly observed in the crude product. By ${ }^{13} \mathrm{C}$ NMR spectroscopy with the mixture of the crude product and authentic sample of diphenyldiselenide, less than $0.5 \%$ of diphenyldiselenide was difficult to detect, if it exists in the reaction mixture. Small peaks between $\mathrm{C} 1$ and $\mathrm{T} 1$ in Figure 3 were assigned as couplings between $\mathrm{Cl}$ and ${ }^{77} \mathrm{Se}$ or $\mathrm{T} 1$ and ${ }^{77} \mathrm{Se}\left(J_{\mathrm{C} 1-\mathrm{Se}}=98.7 \mathrm{~Hz}, J_{\mathrm{T} 1-\mathrm{Se}}=\right.$ $98.6 \mathrm{~Hz}$ ), since the coupling constants of ${ }^{13} \mathrm{C}$ 


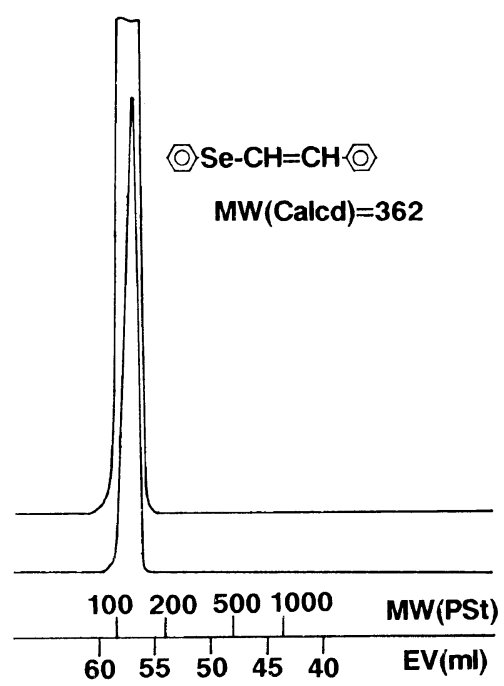

Figure 2. MWD of the crude product in the addition reaction of BSe to EB at $60^{\circ} \mathrm{C}$ in the dark for $2 \mathrm{~h}$ under nitrogen atmosphere. $[\mathrm{EB}]_{0}=[\mathrm{BSe}]_{0}=1.0 \mathrm{moll}^{-1},[\mathrm{AIBN}]_{0}=$ $1.0 \times 10^{-2} \mathrm{moll}^{-1}$ in benzene.

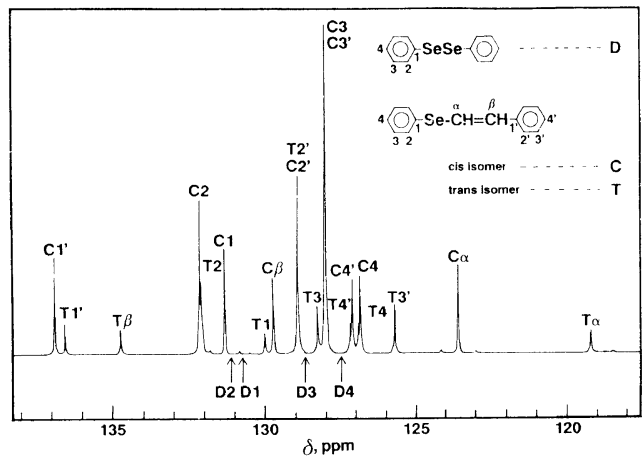

Figure 3. $100 \mathrm{MHz}{ }^{13} \mathrm{C}$ NMR spectrum of the crude product for the addition reaction of BSe to $\mathrm{EB}$ in $\mathrm{CDCl}_{3}$. Chemical shift of diphenyldiselenide measured by ${ }^{13} \mathrm{C}$ NMR: $\delta$ (ppm) 130.8 (D1), 131.1 (D2), 128.7 (D3), 127.5 (D4).

and ${ }^{77} \mathrm{Se}$ of $\mathrm{C} 1$ and $\mathrm{T} 1$ in the adduct approximately agreed with $J_{\mathrm{C}-\mathrm{Se}}=97.8 \mathrm{~Hz}^{16}$ in phenyl vinyl selenide ( $(\mathrm{O})-\mathrm{Se}-\mathrm{CH}=\mathrm{CH}_{2}$ ).

The product was also analyzed by ${ }^{1} \mathrm{H}$ NMR. The ${ }^{1} \mathrm{H}$ NMR spectrum of the crude product shows signals at $\delta \quad 6.70-6.92 \mathrm{ppm}$ due to cis-vinylene protons $\left[J_{\text {cis }}=10.2 \mathrm{~Hz}\left(J_{\text {cis }}=10.4\right.\right.$ $\left.\left.\mathrm{Hz}^{17}\right)\right]$, at $\delta 6.79-7.15 \mathrm{ppm}$ due to transvinylene protons $\left[J_{\text {trans }}=15.3 \mathrm{~Hz}\left(J_{\text {trans }}=16.1\right.\right.$

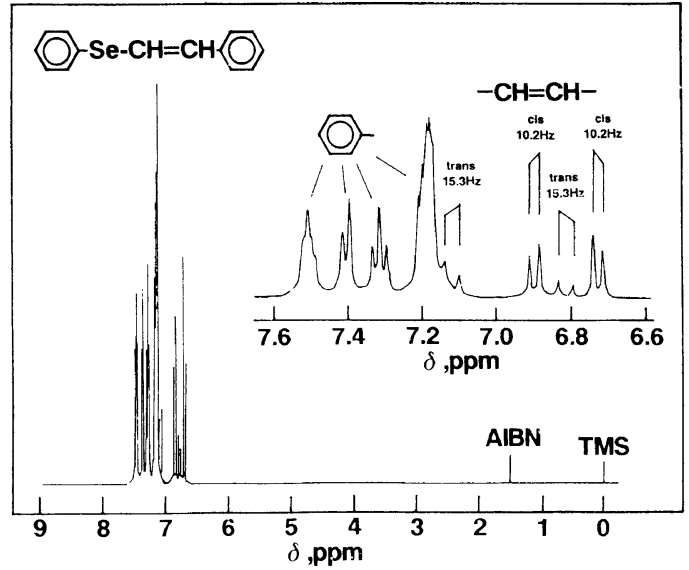

Figure 4. $400 \mathrm{MHz}{ }^{1} \mathrm{H}$ NMR spectrum of the crude product for the addition reaction of $\mathrm{BSe}$ to $\mathrm{EB}$ in $\mathrm{CDCl}_{3}$.

$\left.\left.\mathrm{Hz}^{17}\right)\right]$, and at $\delta 7.11-7.58 \mathrm{ppm}$ due to phenyl ring protons as shown in Figure 4. The spectrum indicates the anti-Markownikoff's structure of the adduct and the ratio of $c i s$ and trans isomers is $75: 25$. The structure was also confirmed by cis- and trans-vinylene absorptions at 1340 and $940 \mathrm{~cm}^{-1}$, respectively, in IR spectrum with liquid method. AIBN may facilitate $c i s \rightarrow$ trans isomerization of the adduct at high temperature. cis Isomer was obtained with $c a .100 \%$ by $\mathrm{BSe}: \mathrm{EB}=1: 1$ in the bulk without AIBN at room temperature under nitrogen atmosphere. ${ }^{18}$

The addition reaction preferentially occurred without appreciable side reaction, and antiMarkownikoff's structure and high cis linkage are consistent with those of the polymer of BDSe and DEB. Accordingly, the addition reaction of BSe to EB can be utilized for kinetic investigation as a model for the addition polymerization of BDSe to DEB.

iii) Reaction Order and Rate-Determining Step. In the kinetic investigation of the model reaction, the reaction order and rate-determining step on the addition reaction were determined. Such kinetic investigation has rarely been done.

The addition reaction of $\mathrm{BSe}$ to $\mathrm{EB}$ is considered to progress by a chain mechanism similar to that of BT to EB. ${ }^{11}$ The elementary 
reactions can be expressed as follows: Initiation Step

$$
\begin{gathered}
\operatorname{AIBN} \stackrel{k_{\mathrm{d}}}{\longrightarrow} 2 \mathrm{R} \cdot+\mathrm{N}_{2} \\
\mathrm{R} \cdot+\bigcirc-\mathrm{SeH} \stackrel{k_{\mathrm{i}}}{\longrightarrow}-\mathrm{Se} \cdot+\mathrm{RH}
\end{gathered}
$$

\section{Addition Step}

$$
\begin{aligned}
& \text { (O) }-\mathrm{Se} \cdot+\mathrm{HC} \equiv \mathrm{C}\left\langle\bigcirc \stackrel{k_{\mathrm{a}}}{\longrightarrow}\right. \\
& \text { (ㄱ) } \mathrm{Se}-\mathrm{CH}=\dot{\mathrm{C}}-\langle\bigcirc
\end{aligned}
$$

Chain Transfer Step

$$
\begin{aligned}
& \text { (O) }-\mathrm{Se}-\mathrm{CH}=\dot{\mathrm{C}}-\bigcirc \mathrm{O}+\mathrm{O}-\mathrm{SeH} \stackrel{k_{\mathrm{tr}}}{\longrightarrow} \\
& \text { (O) }-\mathrm{Se}-\mathrm{CH}=\mathrm{CH}-\mathrm{O}\rangle+(\mathrm{O}-\mathrm{Se} \text {. }
\end{aligned}
$$
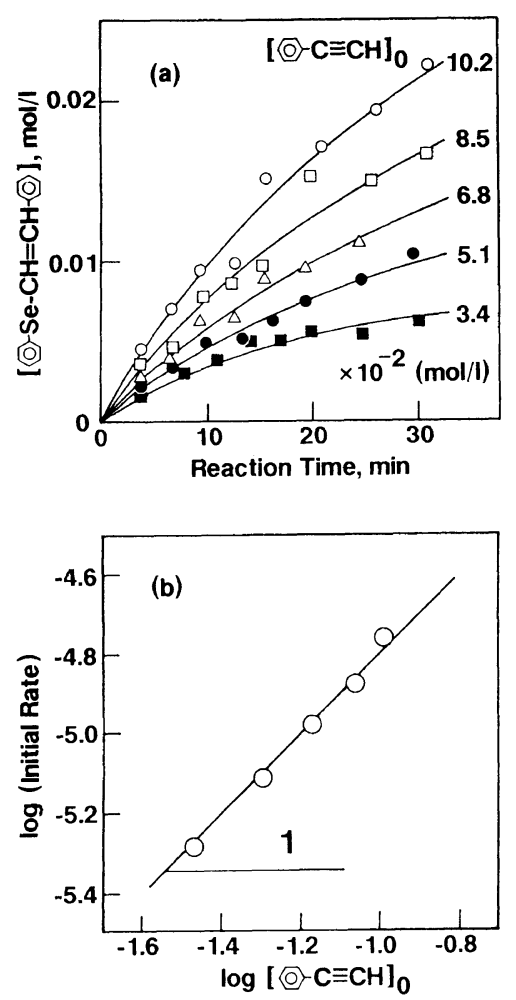

Figure 5. Time-yield curves (a) for the adduct formation of BSe and $\mathrm{EB}$ by changing the initial concentration of $\mathrm{EB}$ and reaction order (b) of $\mathrm{EB}$ at $65^{\circ} \mathrm{C}$ in the dark under nitrogen atmosphere. $[\mathrm{BSe}]_{0}=9.0 \times 10^{-2} \mathrm{moll}^{-1}$, $[\mathrm{AIBN}]_{0}=5.4 \times 10^{-4} \mathrm{moll}^{-1}$ in benzene, int. ref. for GC: $n$-hexadecane. where, $k_{\mathrm{d}}, k_{\mathrm{i}}, k_{\mathrm{a}}$, and $k_{\mathrm{tr}}$ are reaction rate constant for the elementary reactions. Termination, di-addition, and polymerization reactions may take place. However, they are omitted from here, since they were not experimentally detected.

The reaction order of the addition reaction of BSe to EB was determined by a similar method to that in previous papers. ${ }^{1,19}$ Typical time-yield curves and reaction order of EB are shown in Figure 5(a) and (b), respectively, indicating that the addition rate depends on the first order of EB concentration. As shown in Figure 6 the addition rate was independent of BSe concentration. The kinetic order of AIBN was determined as approximately 0.5 (Figure 7), indicating that the addition reaction
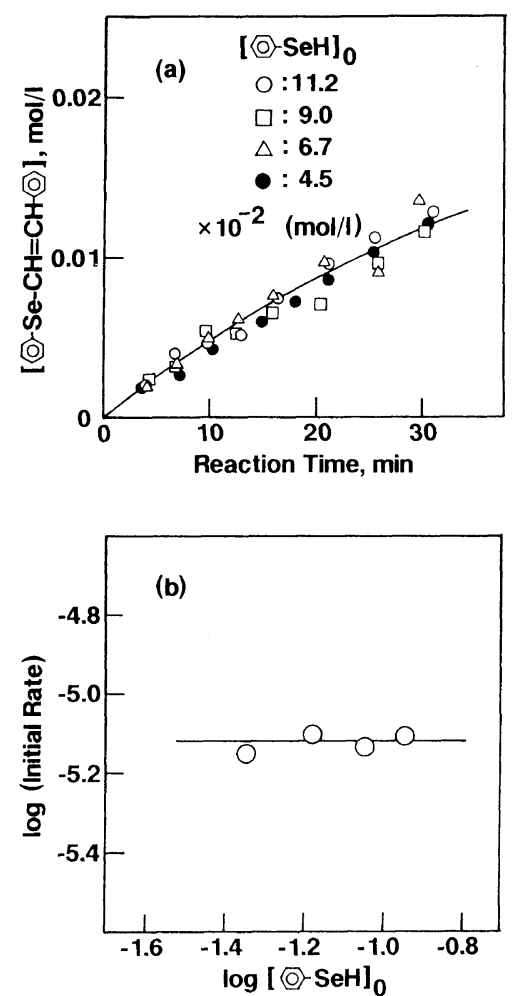

Figure 6. Time-yield curves (a) for the adduct formation of BSe and EB by changing the initial concentration of $\mathrm{BSe}$ and reaction order (b) of $\mathrm{BSe}$ at $65^{\circ} \mathrm{C}$ in the dark under nitrogen atmosphere. $[\mathrm{EB}]_{0}=6.8 \times 10^{-2}$ moll ${ }^{-1},[\mathrm{AIBN}]_{0}=5.4 \times 10^{-4} \mathrm{moll}^{-1}$ in benzene, int. ref. for GC: $n$-hexadecane. 

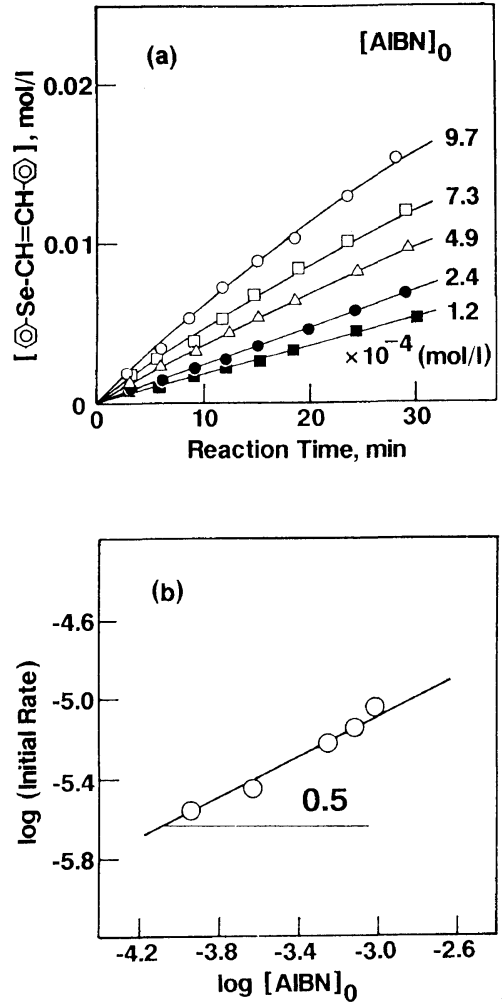

Figure 7. Time-yield curves (a) for the adduct formation of BSe and EB by changing the initial concentration of AIBN and reaction order (b) of AIBN at $65^{\circ} \mathrm{C}$ in the dark under nitrogen atmosphere. $[\mathrm{EB}]_{0}=6.9 \times 10^{-2} \mathrm{moll}^{-1}$, $[\mathrm{BSe}]_{0}=7.8 \times 10^{-2} \mathrm{moll}^{-1}$ in benzene, int. ref. for GC: $n$-hexadecane.

progresses by a radical chain mechanism. Thus, the reaction rate is expressed by eq 8 as,

$$
\text { Rate }=k\left[(\mathrm{O}-\mathrm{C} \equiv \mathrm{CH}]^{1}[\mathrm{O}-\mathrm{SeH}]^{0}[\mathrm{AIBN}]^{0.5}\right.
$$

where $k$ is the overall reaction rate constant at $65^{\circ} \mathrm{C}$ in benzene. On comparing eq 8 with a theoretical rate equation, ${ }^{11}$ the rate-determining step was shown to be the addition step of the benzeneseleno radical to EB.

\section{Comparison between BSe and BT or EB and St}

i) Comparison between $B S e$ and $B T$ in the Addition Reaction of $E B$. The results of the addition reaction of $\mathrm{BSe}$ or $\mathrm{BT}^{11}$ to $\mathrm{EB}$ are summarized in Table I. BSe and BT add to EB without appreciable side reaction. The adducts
Table I. Comparison of addition reactions of BSe or BT to EB

\begin{tabular}{|c|c|c|}
\hline & (ㄱ) $\mathrm{SeH}$ & (O) $-\mathrm{SH}^{11}$ \\
\hline Yield/\% & $\simeq 100^{\mathrm{a}}$ & $\simeq 100^{\mathrm{a}}$ \\
\hline Structure & $\begin{array}{c}\text { Anti- } \\
\text { Markownikoff }\end{array}$ & $\begin{array}{c}\text { Anti- } \\
\text { Markownikoff }\end{array}$ \\
\hline $\begin{array}{l}\text { Reaction order } \\
{[\text { AIBN] }}\end{array}$ & 0.5 & 0.5 \\
\hline $\begin{array}{l}{[\langle\mathrm{O}\rangle \mathrm{SeH}]} \\
\text { or }[\mathrm{O}-\mathrm{SH}]\end{array}$ & 0 & 0 \\
\hline$[$ (O) $\mathrm{C} \equiv \mathrm{CH}]$ & 1 & 1 \\
\hline $\begin{array}{l}\text { Rate-determining } \\
\text { step }\end{array}$ & Addition & Addition \\
\hline $\begin{array}{l}{[\mathrm{BSe}]_{0}=[\mathrm{BT}]_{0}=} \\
10^{-2} \mathrm{moll}^{-1} \text { in } \\
2 \mathrm{~h} \text { under nitrog }\end{array}$ & $\begin{array}{l}\mathrm{B}]_{0}=1 \mathrm{moll}^{-1} \text {; } \\
\text { nzene at } 60^{\circ} \mathrm{C}\end{array}$ & $\begin{array}{l}{[\mathrm{AIBN}]_{0}=1.0 \times} \\
\text { in the dark for }\end{array}$ \\
\hline
\end{tabular}

of the anti-Markownikoff's structure were obtained in nearly $100 \%$ yield. The addition reactions of $\mathrm{BSe}$ or $\mathrm{BT}$ to $\mathrm{EB}$ have the same reaction orders; that is, the rate-determining step is the addition step of the benzeneseleno radical (see eq 6) or the benzenethiyl radical to EB. In the addition reaction of BT to styrene $(\mathrm{St})^{19}$ or addition reactions of diphenylphosphine to $\mathrm{St}^{20}$ and $\mathrm{EB},{ }^{21}$ the rate-determining step was the chain transfer step between the intermediate carbon radicals and BT or diphenylphosphine. According to quantum chemical calculation by CAChe system, the rate-determining step was expected to be the chain transfer step in the addition reaction of BT to EB. ${ }^{22}$ However, the rate-determining step was experimentally confirmed to be the addition step. ${ }^{11}$ The addition reaction of BT to EB may be affected by CT interaction between BT and EB. ${ }^{23}$ Thus the chain transfer step of the reaction may occur very rapidly as a sort of cage effect due to CT interaction. CT interaction between $\mathrm{BSe}$ and $\mathrm{EB}$ was also supported by diffuse reflectance spectrum of a 
thin layer mixture of BDSe and DEB. ${ }^{10}$ Consequently, it is considered that CT interaction between BT and EB or between BSe and $E B$ affects the rate-determining step.

ii) Comparison between EB and St in the Addition Reaction of $B S e$. The results of the addition reaction of $\mathrm{BSe}$ to $\mathrm{EB}$ or $\mathrm{St}^{12}$ are summarized in Table II. In the addition reaction of BSe to St, the adduct was obtained in $62 \%$ yield. The low yield was caused by termination reaction (eq 9) and side reaction (eq 10). AIBN was not used effectively for the addition reaction.

$$
\begin{aligned}
& 2\langle\mathrm{O}-\mathrm{Se} \cdot \longrightarrow \text { O }-\mathrm{Se}-\mathrm{Se}-\mathrm{O}
\end{aligned}
$$

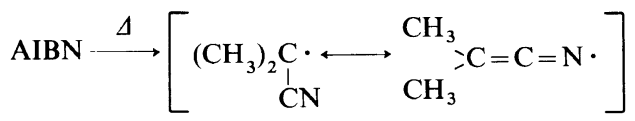

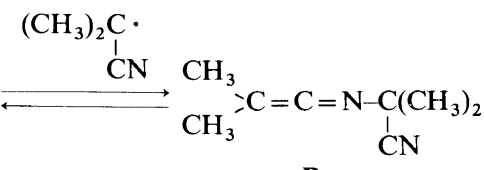

$$
\begin{aligned}
& \text { B } \\
& \stackrel{\text { (O)-Se }}{\longrightarrow} \underset{\mathrm{H} \cdot}{\longrightarrow} \begin{array}{c}
\left(\mathrm{CH}_{3}\right)_{2} \mathrm{CH} \\
\left(\mathrm{CH}_{3}\right)_{2}{ }_{\mathrm{C}}^{\mathrm{C}-\mathrm{N}}
\end{array}=\mathrm{C}-\mathrm{Se}-\mathrm{O}
\end{aligned}
$$

The structures of the adducts were the anti-Markownikoff's type, and the addition reactions of $\mathrm{BSe}$ to $\mathrm{EB}$ or St have the same reaction orders. That is, the rate-determining step is the addition step of the benzeneseleno radical to EB or St. On the contrary, addition reactions of BT to EB and St have different reaction orders. The rate-determining step is the addition step in the addition reaction of BT to EB, and is the chain transfer step in that of BT to St. The hydrogen abstraction from BSe occurs much more rapidly in comparison with that from BT. In former case the addition step becomes the rate-determining step. The reactivity of hydrogen abstraction of cyclopropylcarbinyl radical from BSe and BT was
Table II. Comparison of addition reactions

\begin{tabular}{|c|c|c|}
\hline & \multicolumn{2}{|c|}{ () $-\mathrm{C} \equiv \mathrm{CH}$ (O) $\mathrm{CH}=\mathrm{CH}_{2}{ }^{12}$} \\
\hline Yield $/ \%$ & $\simeq 100^{\mathrm{a}}$ & $62^{b}$ \\
\hline Structure & $\begin{array}{c}\text { Anti- } \\
\text { Markownikoff }\end{array}$ & $\begin{array}{c}\text { Anti- } \\
\text { Markownikoff }\end{array}$ \\
\hline $\begin{array}{l}\text { By-product } / \% \\
\text { diphenyldiselenide } \\
\text { compound } \mathbf{A}^{\mathrm{c}}\end{array}$ & $\begin{array}{l}\text { none } \\
\text { none }\end{array}$ & $\begin{array}{r}1.9 \\
\simeq 0.1\end{array}$ \\
\hline
\end{tabular}
of BSe to EB or St

\begin{tabular}{|c|c|c|}
\hline $\begin{array}{l}\text { Reaction order } \\
\text { [AIBN] }\end{array}$ & 0.5 & 0.5 \\
\hline$[\langle-\mathrm{SeH}]$ & 0 & 0 \\
\hline $\begin{array}{l}{[\mathrm{O}-\mathrm{C} \equiv \mathrm{CH}]} \\
\text { or }\left[\bigcirc-\mathrm{CH}=\mathrm{CH}_{2}\right]\end{array}$ & 1 & 1 \\
\hline $\begin{array}{l}\text { Rate-determining } \\
\text { step }\end{array}$ & Addition & Addition \\
\hline \multicolumn{3}{|c|}{$\begin{array}{l}\text { a See Table I. } \\
\text { b }[\mathrm{BSe}]_{0}=[\mathrm{St}]_{0}=2 \mathrm{moll}^{-1} ;[\mathrm{AIBN}]_{0}=1.5 \times 10^{-2} \mathrm{mo} \\
1^{-1} \text { in benzene at } 70^{\circ} \mathrm{C} \text { in the dark for } 19 \mathrm{~h} \text { under } \\
\text { nitrogen atmosphere. } \\
\text { c See eq } 10 .\end{array}$} \\
\hline
\end{tabular}

\section{$\mathrm{BSe} / \mathrm{BT} \simeq 15$ in $\mathrm{THF}$ at $25^{\circ} \mathrm{C}^{24}$}

iii) Relative Reactivity of $B S e$ and $B T$. Though selenol compounds as well as thiol compounds add to unsaturated bonds, ${ }^{25-29}$ the relative reactivities of selenol and thiol compounds to unsaturated bonds in the radical addition have been studied little. Previously, the authors reported the competitive addition reactions of BSe and BT to St, and the results indicated that the reactivity of BSe was much higher than BT. ${ }^{12}$

The authors investigated the reactivity between selenol and thiol compounds to EB. The relative reactivity was determined by competitive addition reactions of $\mathrm{BSe}$ and BT to EB without AIBN. A trace amount of oxygen in the reaction vessel may also initiate the reaction. Figure 8 shows the time-yield curves of the adduct formation. EB reacts with 


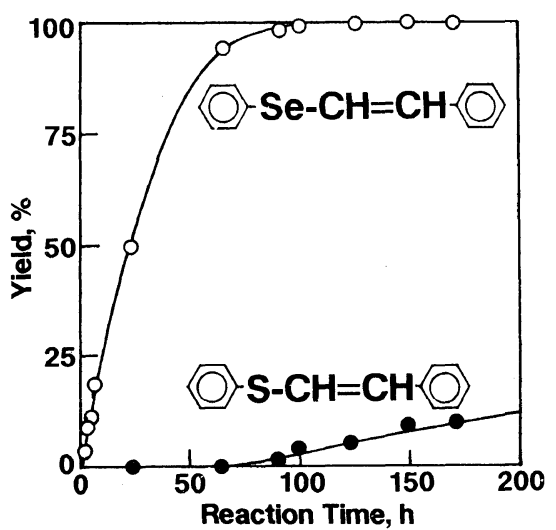

Figure 8. Competitive addition reaction of BSe $(O)$ and $\mathrm{BT}(\mathrm{O})$ to $\mathrm{EB}$ at $34^{\circ} \mathrm{C}$ in the dark under nitrogen atmosphere. $[\mathrm{EB}]_{0}=4.2 \mathrm{moll}^{-1},[\mathrm{BSe}]_{0}=[\mathrm{BT}]_{0}=2.1 \mathrm{moll}^{-1}$ in toluene (by ${ }^{1} \mathrm{H}$ NMR).

BSe preferentially at first, and then the addition reaction of $\mathrm{BT}$ to $\mathrm{EB}$ starts when $\mathrm{BSe}$ is almost consumed. The benzeneseleno radical is created preferentially in the reaction mixture, since the bond energy of $\mathrm{Se}-\mathrm{H}\left(<73 \mathrm{kcal} \mathrm{mol}^{-114}\right)$ is smaller than that of S-H $\left(82 \mathrm{kcal} \mathrm{mol}^{-130}\right)$. The benzenethiyl radical in the reaction mixture produces the benzeneseleno radical and BT by reaction with BSe. That is, the hydrogen transfer reaction between the benzenethiyl radical and BSe occurs. The process could not be confirmed by conventional experiment, since D-H exchange between $-\mathrm{SeD}$ and - $\mathrm{SH}$ occurred even in the presence of tetraphenylphosphonium bromide electrolyte. However, $\Delta G\left(-16.8 \mathrm{kcal} \mathrm{mol}^{-1}\right)$ evaluated by CAChe system indicates that the equilibrium constant $K$ is $10^{12}$.

$$
\text { (O) }-\mathrm{SeH}+(\mathrm{O}-\mathrm{S} \cdot \longrightarrow \mathrm{O}-\mathrm{Se} \cdot+\mathrm{O}-\mathrm{SH}
$$

$K$ is compatible with the idea that the benzeneseleno radical exists preferentially in the reaction mixture.

iv) Relative Reactivity of EB and St. Figure 9 shows time-yield curves of the adduct formation of BSe with EB and St. BSe adds to EB much more rapidly than St. EB has 80 times the high reactivity of $\mathrm{St}$. The reactivity means the relative rate of addition step of benzene-

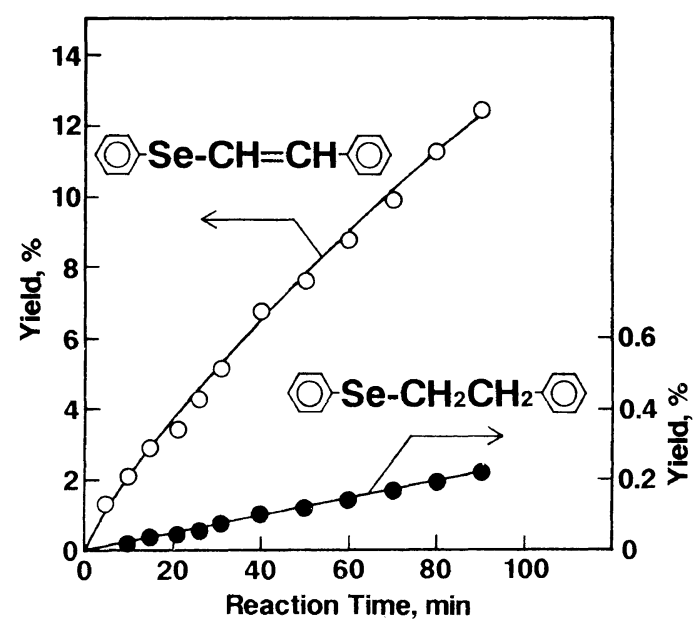

Figure 9. Competitive addition reaction of BSe to EB $(\mathrm{O})$ and $\mathrm{St}(\mathrm{O})$ at $60^{\circ} \mathrm{C}$ in the dark under nitrogen atmosphere. $[\mathrm{BSe}]_{0}=7.0 \times 10^{-2} \mathrm{moll}^{-1},[\mathrm{~EB}]_{0}=[\mathrm{St}]_{0}=$ $6.6 \times 10^{-2} \mathrm{moll}^{-1},[\mathrm{AIBN}]_{0}=3.4 \times 10^{-4} \mathrm{moll}^{-1}$ in benzene, int. ref. for GC: $n$-hexadecane.

seleno radical to $\mathrm{EB}$ and $\mathrm{St}$, since these reactions have the same rate-determining addition step. In the addition reaction of $\mathrm{BSe}$ to $\mathrm{St}$, diphenyldiselenide with $1.9 \%$ and compound A with $0.1 \%$ were confirmed. ${ }^{12}$ The formation of by-products implies that the rate of the addition reaction of benzeneseleno radical to $\mathrm{St}$ is not so high and benzeneseleno radicals have a chance to dimerize (eq 9) and form compound $\mathbf{A}$ by reaction with $\mathbf{B}$ in eq 10 . The by-products were not detected in the addition reaction of $\mathrm{BSe}$ to $\mathrm{EB}$. The benzeneseleno radical added to $E B$ much more rapidly than St. High reactivity of EB toward benzeneseleno radical suppressed the formation of diphenyldiselenide and compound $\mathbf{A}$.

\section{REFERENCES}

1. K. Murata, T. Ukachi, H. Anzai, G. Saito, K. Kajimura, and T. Ishiguro, J. Phys. Soc. Jpn., 51, 1817 (1982).

2. D. J. Sandman, M. Rubner, and L. Samuelson, J. Chem. Soc., Chem. Commun., 1133 (1982).

3. M. A. Druy and R. J. Seymour, J. Phys. Paris Colloq., C3, 595 (1983).

4. E. Kobayashi, T. Ohashi, and J. Furukawa, Makromol. Chem., 187, 2525 (1986). 
5. E. Kobayashi, Y. Ishizuka, Y. Kochaku, and J. Furukawa, Kobunshi Ronbunshu, 46, 203 (1988).

6. E. Kobayashi, J. Jiang, S. Aoshima, and J. Furukawa, Polym. J., 22, 146 (1990).

7. E. Kobayashi, T. Ohashi, and J. Furukawa, J. Polym. Sci., A, Polym. Chem., 25, 2077 (1987).

8. E. Kobayashi, T. Ohashi, and J. Furukawa, Polym. Adv. Technol., 2, 301 (1991).

9. E. Kobayashi, N. Metaka, S. Aoshima, and J. Furukawa, J. Polym. Sci., A, Polym. Chem., 30, 227 (1992).

10. E. Kobayashi, N. Metaka, S. Aoshima, and J. Furukawa, Polym. Prepr. Jpn., 39, 1648 (1990).

11. E. Kobayashi, T. Obata, S. Aoshima, and J. Furukawa, Polym. J., 22, 1035 (1990).

12. E. Kobayashi, K. Takehara, and S. Aoshima, Kobunshi Ronbunshu, 51, 337 (1994).

13. G. Foster, Org. Synth., Collect. Vol. III, 771 (1955).

14. T. Masawaki, Y. Uchida, A. Ogawa, N. Kambe, N. Miyoshi, and N. Sonoda, J. Phys. Org. Chem., 1, 115 (1988).

15. A. Fave, G. Reichenbach, and U. Peron, J. Am Chem. Soc., 89, 6696 (1967).

16. G. A. Kalabin and D. F. Kushnarev, Zh. Struk. Khim., 20, 617 (1979).

17. H. J. Cristau, B. Chabaud, R. Labaudiniere, and H. Christol, J. Org. Chem., 51, 878 (1986).
18. E. Kobayashi, N. Metaka, S. Aoshima, and J. Furukawa, Polym. Prepr. Jpn., 38, 1581 (1989).

19. E. Kobayashi, T. Obata, S. Aoshima, and J. Furukawa, Polym. J., 22, 803 (1990).

20. E. Kobayashi, T. Obata, S. Aoshima, and J. Furukawa, Polym. J., 25, 1039 (1993).

21. E. Kobayashi, T. Obata, S. Aoshima, and J. Furukawa, Polym. J., 25, 1049 (1993).

22. T. Obata, E. Kobayashi, S. Aoshima, and J. Furukawa, Polym. J., 24, 757 (1992).

23. E. Kobayashi, Y. Terada, T. Ohashi, and J. Furukawa, Polym. J., 23, 267 (1991).

24. M. Newcomb and M. B. Manek, J. Am. Chem. Soc., 112, 9662 (1990).

25. F. G. Gabdrakhmanov, Yu. Yu. Samitov, and E. G. Kataev, Zh. Obshch. Khim., 37, 761 (1967).

26. T. Masawaki, A. Ogawa, N. Kambe, I. Ryu, and N. Sonoda, Chem. Lett., 2407 (1987).

27. J. Gosselk, Angew. Chem., Int. Ed. Engl., 2, 660 (1963).

28. E. G. Kataev and V. N. Petrov, Zh. Obshch. Khim., 32, 3699 (1962).

29. A. Ogawa, H. Yokoyama, K. Yokoyama, T. Masawaki, N. Kambe, and N. Sonoda, J.Org. Chem., 56, 5721 (1991).

30. S. W. Benson, Chem. Rev., 78, 23 (1978). 